\title{
Fluorescence-based Techniques to Study the Structure and Dynamics of Mass- selected Biomolecular Ions
}

\author{
Prince Tiwari* \\ SCS-Metrohm award for best oral presentation in Analytical Sciences
}

\begin{abstract}
Laser-induced fluorescence studies on mass-selected biomolecules are a promising route to understand their properties in the gas phase and probe their intrinsic properties in a solvent-free environment. Fluorescence has been used to investigate the conformation and dynamics of gaseous biomolecular ions. With Förster Resonance Energy Transfer (FRET), it is now possible to obtain sensitive intramolecular distance information from large biomolecules, like proteins, with high chemical specificity. With growing interest and applications, gas-phase fluorescence measurements can shed greater light on the characteristics of proteins in the gas phase. Compared to the solution phase measurements, gas-phase fluorescence can also help understand the influence of solvent interactions on the protein structure and function.
\end{abstract}

Keywords: Biomolecular structure · Dynamics · Fluorescence · Ion spectroscopy · Mass spectrometry

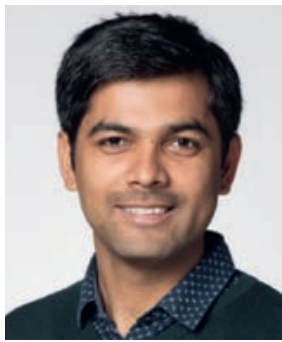

Prince Tiwari completed his Integrated Master of Science (Chemistry) from the National Institute of Science Education and Research (India) as an INSPIRE fellow. He investigated the formation of organic molecules in the circumstellar disc of a carbon star for his master's thesis in the group of Dr. H. S. Biswal. While pursuing his PhD in the group of Prof. Renato Zenobi at ETH Zürich, Prince has developed different experimental setups and techniques to enhance fluorescence measurements of trapped, mass-selected biomolecular ions in the gas phase. He likes to run, hike, and follow technological developments of his interest in his free time.

\section{Gas-phase Fluorescence Spectroscopy of Biomolecular lons}

Laser-induced fluorescence (LIF) is an established and widely used tool to probe the conformation and dynamics of biomolecules in the solution phase. ${ }^{[1]}$ Combined with the selectivity provided by mass spectrometry (MS), LIF techniques can also provide detailed insight into the intrinsic properties of gaseous biomolecules in well-controlled environments. ${ }^{[2-6]}$ Structural investigations of biomolecules in the gas phase have several advantages. By comparing with the solution-phase measurements, gas-phase studies can help us better understand the role of solvent interactions on protein behavior. They also facilitate the answer to hotly debated questions like whether the gaseous biomolecules retain their solution-phase structures or not. The measurements and constraints obtained from gaseous biomolecules also act as a valuable benchmark for molecular modeling. ${ }^{[7]}$

LIF and MS have individually proved very useful for the analysis of the biomolecules. ${ }^{[1,8]} \mathrm{MS}$ and allied techniques offer many key capabilities to study various biomolecules like sugar, proteins, and oligonucleotides. These capacities have further benefitted from the introduction of native electrospray ionization, enabling the transfer of biomolecular ions to the gas phase in native-like conformations. ${ }^{[9]}$ The accurate mass information obtained from a mass spectrum allows identification of a protein, its complexes, any post- or co-translational modifications in it, or heterogeneities present in the sample. Tandem MS capabilities enable high chemical specificity as ions of a specified $\mathrm{m} / \mathrm{z}$, representing a charge state, an oligomer, or a complex stoichiometry can be easily isolated for further analysis (demonstrated in the right panel of Fig. 1a). MS-related techniques like H-D exchange, cross-linking and ion mobility spectrometry are widely used to probe biomolecular conformers in the gas phase. ${ }^{[10]}$

Fluorescence studies of gaseous biomolecular ions can provide orthogonal information compared to those obtained from MS-based techniques, enabling a more detailed insight into the structure and dynamics of biomolecules in the gas phase. When coupled with MS, it is possible to perform fluorescence experiments on a pre-selected ion population and remove everything else, which significantly simplifies the data analysis. Fluorescence-based techniques like FRET can help obtain intramolecular distances between two selected points within a biomolecule. The intramolecular distances can give insight into different conformations a biomolecule can have, which is highly relevant for molecular modeling. A similar technique known as dynamic fluorescence quenching can be used to study the protein dynamics and stability by probing local fluctuations. ${ }^{[3,4]}$

The gas-phase fluorescence measurements can provide promising results but are challenging to perform. The significant challenges in gas-phase fluorescence measurements are low fluorescence signal, unknown photophysics of fluorophores in the gas-phase, and the need to couple an optical excitation and detection system with an ion trap mass spectrometer. ${ }^{[11,12]}$ Nonetheless, some research groups have risen to the challenge and performed remarkable ex- 
periments. ${ }^{[11,13-16]}$ Fluorescence from trapped gaseous ions was first reported way back in 1981.[17] Fluorescence from trapped fluorescent dyes could only be obtained in the early 2000s. ${ }^{[18,19]}$ Soon after, fluorescence-based techniques were used to probe conformers, dynamics, and structural heterogeneities in mass-selected biomolecules like oligonucleotides, polyprolines, and proteins. ${ }^{[2,5,20]}$ Here, we present some key advances in the study of gaseous biomolecules using fluorescence-based techniques.

\section{Platform for Diverse Gas-phase Fluorescence Measurements with High Fluorescence Collection Efficiency}

One of the critical challenges in the gas-phase fluorescence measurements is the low signal intensity. The low signal stems from low ion density ( fM) in the ion trap and limited fluorescence collection solid angle because of structural constraints of the mass spectrometer. The absence of solvent molecules to effectively dissipate heat after laser excitation leads to high photo-fragmentation of molecular ions and limits the range of laser power that can be used $(\mu \mathrm{W}-\mathrm{mW})$, further resulting in a weak signal. The use of ion traps allows a prolonged exposure of ions with a laser beam, and addition of multiple fluorescence acquisitions make it possible to perform fluorescence measurements of scarce ion population. Thus, ion trap mass spectrometers are the obvious choice for coupling optics for fluorescence measurements. Out of a handful of gas-phase fluorescence measurement setups that have been developed, some provide high fluorescence collection efficiency but are limited in the types of experiments that can be performed. Some setups can perform a variety of experiments but lack high fluorescence collection efficiency.[11,14,21] The diverse experimental functionalities are needed to gain more insights into the protein structure, while high fluorescence collection efficiency helps reduce the experimental times and perform experiments even in low brightness conditions. The high collection efficiency becomes especially critical in the case of biomolecular ions. The multiple charge states and adducts reduce the number of ions of interest in the trap, and the high charge reduces the space-charge limited ion density, leading to a weaker fluorescence signal. We recently developed an instrument in our lab that facilitates diverse fluorescence experiments with high fluorescence collection efficiency. ${ }^{[16]}$ Fluorescence-based experiments like excitation and emission spectroscopy, lifetime measurements, and ion imaging of trapped ions can be performed with this setup. And the high fluorescence collection efficiency results from the large fluorescence collection solid angle that could be achieved with a lens placed very close to the ion cloud.

Key steps in obtaining fluorescence measurement are shown in Fig. 1a. A $\sim \mu \mathrm{M}$ sample of a dye or a biomolecule labeled with a dye is electrosprayed to generate gaseous ions. The ions are transported to the ion trap through the mass spectrometer's ion optics, and ions of the desired $\mathrm{m} / \mathrm{z}$ are isolated, providing mass selection, and the rest ejected. The mass-selected ions are excited with a laser beam of appropriate wavelength and power. The fluorescence emission from the ions is collected through a lens placed in a hole drilled in the ring electrode of the ion trap. The large fluorescence collection solid angle of the lens allows $\sim 2.3 \%$ of emitted fluorescence to be collected. The collected fluorescence emission is directed to a fluorescence detection system where spectrally- and time-resolved fluorescence can be measured using a highly sensitive, electrothermally-cooled CCD detector and a single-photon avalanche diode detector, respectively.

(a)

\section{Fluorescence}
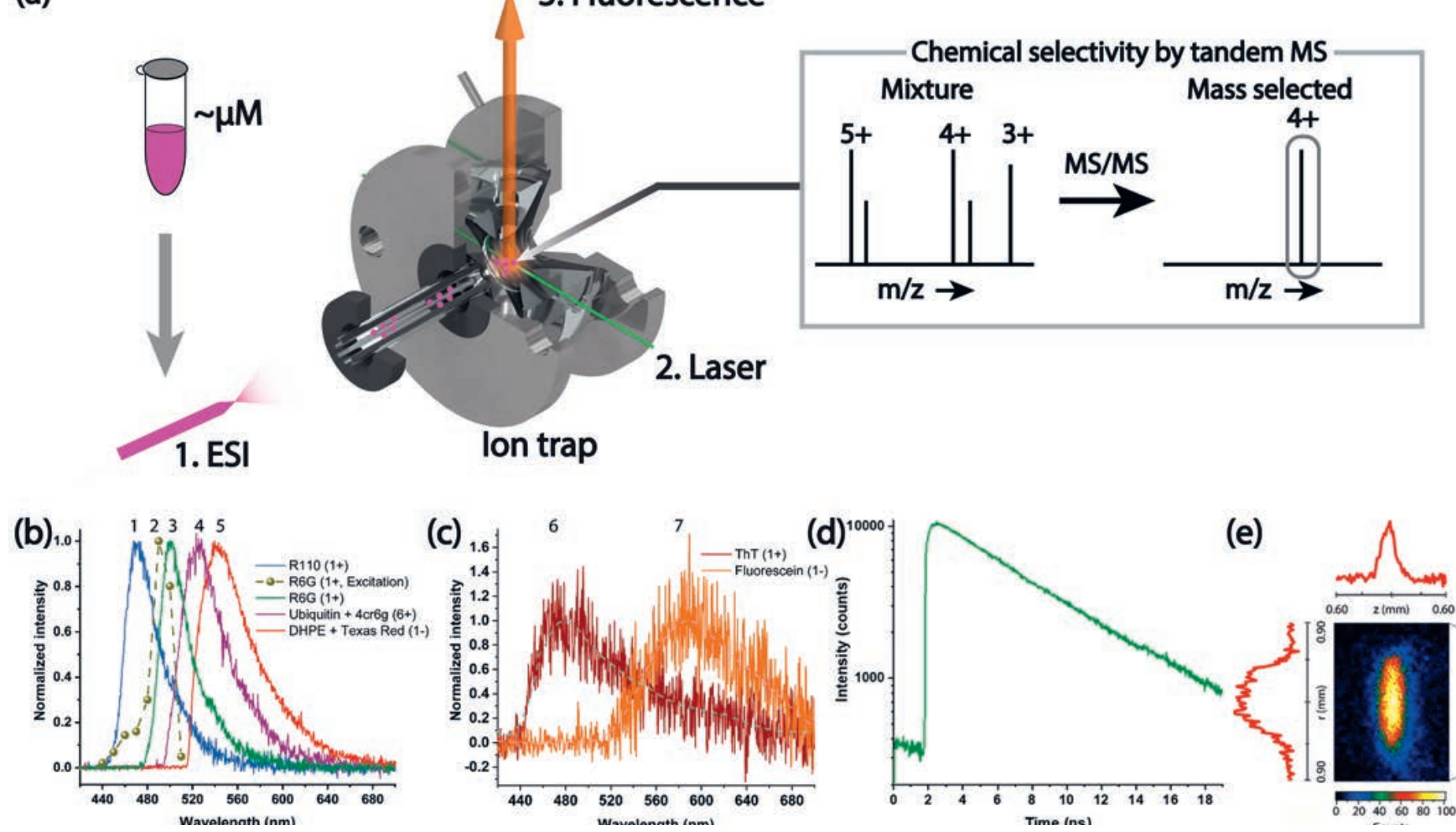

Fig. 1. Gas-phase fluorescence measurements with high collection efficiency: The figure shows key steps in a fluorescence measurement performed on trapped ions and various results that can be obtained. (a) The ions are generated from a $\sim \mu \mathrm{M}$ concentration solution using electrospray ionization. The ions are transported to the ion trap through ion optics of the mass spectrometer and desired $\mathrm{m} / \mathrm{z}$ ions are selected. The trapped ions are irradiated with a laser beam (2), and the emitted fluorescence (3) is collected with the help of a lens embedded in a hole drilled in the ring electrode of the ion trap. The collected fluorescence emission is used for different kinds of fluorescence measurements: (b \& c) excitation and emission spectra of different analytes; (d) fluorescence decay; and (e) false-color image of trapped ions. Figure adapted with permission from the publisher from ref. [16]. 
The characteristic feature of this instrument is diverse fluorescence measurements with high collection efficiency. Figs 1b-e illustrate these capabilities. Fluorescence spectra of ions in a wide range of wavelengths and different sizes are presented along with a fluorescence decay and an ion image. Measurements of bright fluorescent dye ions such as rhodamine $6 \mathrm{~g}$ (R6G) and rhodamine 110 (R110) could be performed in a matter of seconds and for low brightness dye ions such as thioflavin T (ThT) and fluorescein, in a few minutes. Higher signal-to-noise $(\mathrm{S} / \mathrm{N})$ spectra, compared to those in literature, of very low brightness dyes thioflavin $\mathrm{T}$ and fluorescein were also acquired to illustrate the high fluorescence detection capabilities of the instrument. ${ }^{[12,22]}$ Fluorescence spectra of biomolecules like carboxyrhodamine $6 \mathrm{~g}$-labeled ubiquitin and DHPE lipid - Texas red conjugate could be obtained with high $\mathrm{S} / \mathrm{N}$. The fluorescence excitation and emission spectra report a change in the microenvironment of the dye ${ }^{[23]}$ and the fluorescence decay indicates a change in the deactivation pathways and presence of structural heterogeneities. ${ }^{[20]}$ The ion image shows the position of ions within the ion trap. The high fluorescence collection efficiency is vital to study biomolecules. It enables the measurements from less abundant ions in a mixture and from experiments where the fluorescence is partially quenched. It also gives the flexibility to use low quantum yield dyes for probing biomolecular structures.

\section{Distance Probes for Gaseous Biomolecules: FRET and Action-FRET}

Förster Resonance Energy Transfer (FRET) is the radiationless transfer of energy from an electronically excited fluorophore to a nearby acceptor. It is one of the most popular fluorescence-based techniques in the solution phase used to probe the biomolecular structure and monitor conformational changes. It can be a promising addition to the existing methods used to study gas-phase biomolecules as the distance information obtained with FRET cannot be obtained directly with MS-based techniques. The intramolecular distance in the range of 2-10 $\mathrm{nm}$ determined in FRET experiments is highly dependent on the distance and orientation between a donor and an acceptor, strategically labeled to the biomolecule. The distances are measured through FRET efficiency when the energy is non-radiatively transferred from the donor fluorophore to the acceptor. The FRET efficiency can be measured by monitoring the fluorescence emission of the donor and acceptor fluorophores or the donor's fluorescence decay profile.

J. H. Park's lab pioneered the FRET on mass-selected biomolecules in 2003 when they presented the study on a multiply charged oligonucleotide complex by measuring the change in total donor fluorescence intensity. ${ }^{[2]}$ The Zenobi group reported FRET in ultra-high vacuum and identified multiple FRET pairs in the gas phase. ${ }^{[24]}$ Gas-phase FRET from fluorescently labeled polyproline-based peptides was reported by the Jockusch group, who unraveled the intricacies of the polyproline conformers in the gas phase. ${ }^{[5]}$ Recently, gas-phase FRET measurements were used to study conformers of mass-selected protein in different charge states. A mutant variant of the B1 domain of protein $G$ was FRET labeled, and fluorescence emission and lifetime of different charge states were measured. The FRET efficiency decreased with the increasing charge state of the gaseous protein indicating a more extended conformation (Fig. 2a). The estimated inter-dye distances from the FRET measurements also showed that the proteins are more expanded in the gas phase even in a $3+$ charge state than their solution-phase structure. ${ }^{[4]}$ (a)
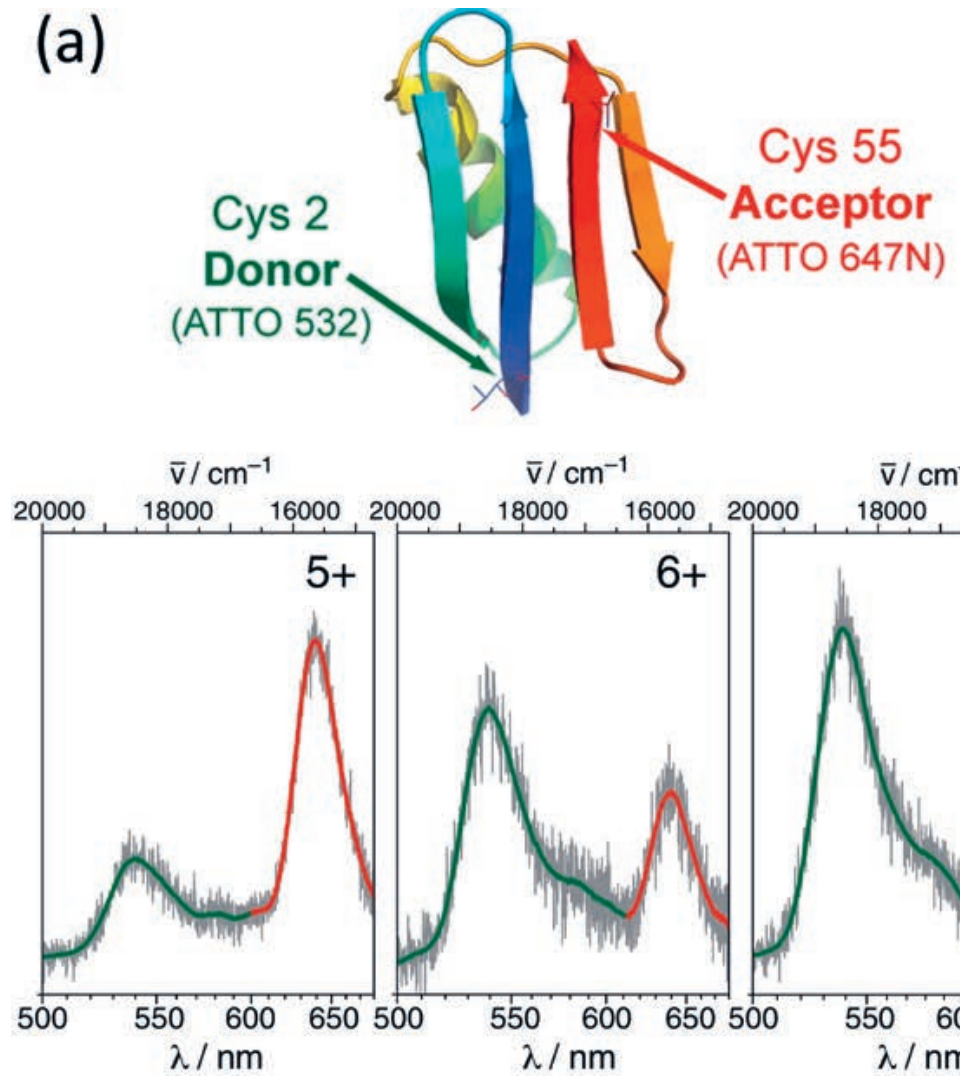

$\overline{\mathrm{v}} / \mathrm{cm}^{-1}$ $18000 \quad 16000$

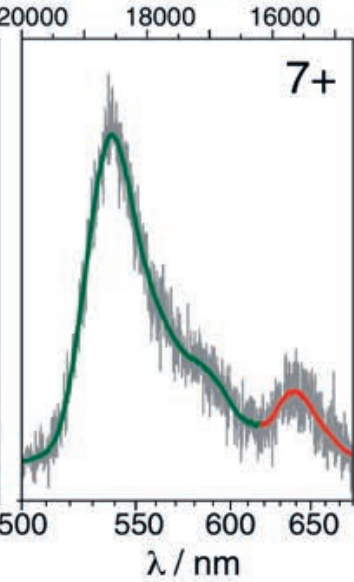

(b)

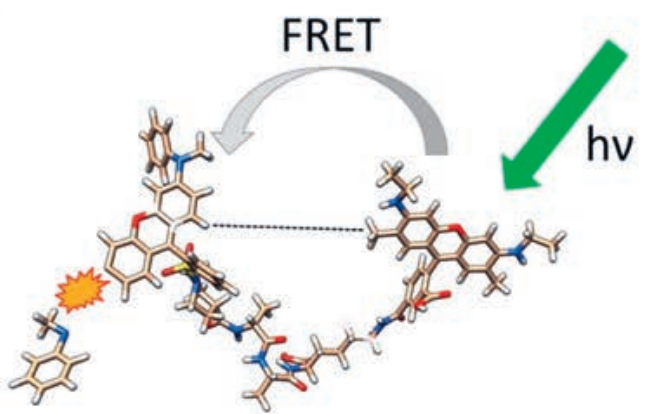

Fig. 2. FRET and action-FRET in the gas phase: (a). FRET obtained from mass-selected GB1 protein in different charge states. The protein was labeled with a FRET pair at Cys 2 and Cys 55. The fluorescence emission with increasing charge states shows the decreasing intensity of acceptor fluorescence (red trace), implying decreasing FRET efficiency and increasing donor-acceptor distances. Adapted with permission from the publisher. ${ }^{20}$ Copyright 2015 American Chemical Society. (b). Peptides with different donor-acceptor distances show decreasing FRET efficiency with increasing donor-acceptor minimum separation. Reproduced with permission from ref. [25]. Copyright 2014 American Chemical Society. 
To perform gas-phase FRET experiments without the need to measure the low fluorescence emission, a new technique by the name of action-FRET was developed. Action-FRET employs an acceptor that can fragment upon non-radiative energy transfer from the donor. The fragment ion intensity, which should be proportional to the FRET efficiency, can be easily monitored in the mass spectrum. In an action-FRET study on a peptide labeled with a FRET pair, it was also shown that the FRET efficiency derived from the action-FRET measurements was monotonically decreasing with increasing donor-acceptor separation (Fig. 2b). ${ }^{[25]}$

FRET in the gas phase can provide critical insights into the biomolecular structure using only picoliters of sample in $\mu \mathrm{M}$ concentration. However, some groundwork still needs to be done before the intramolecular distances can be accurately measured with FRET. To have an accurate estimate, Förster radius $\left(\mathrm{R}_{0}\right)$ and other parameters like the quantum yield of donor and acceptor, the molar absorptivity of the acceptor, and orientation factor $\left(\kappa^{2}\right)$ need to be determined within a reasonable uncertainty. ${ }^{[4]}$ Several studies are being performed to understand the fundamentals of FRET in the gas phase. ${ }^{[26,27]}$ Development of action-FRET can also lead to its wider adaptability as it does not require out-coupling of the fluorescence emission, thus greatly simplifying the experiments.

\section{Probes for Protein Dynamics in the Gas Phase}

Fluorescence can also be a useful tool to study the conformational changes in proteins. Dynamic contact quenching of a fluorophore occurs when a quencher comes in close contact with an excited fluorophore. It is a sensitive probe to conformational changes with evolving interaction between the fluorophore and the quencher within a biomolecule. Park's lab has used this technique to investigate the role of solvation on the dynamics of Trp-cage (a mini-protein) upon thermal denaturation in solution and the gas phase within a temperature-controlled ion trap. ${ }^{[28]}$ BODIPY dye covalently linked to the protein was used as a fluorescent probe, and Trp acted as the quencher. With increasing temperature from $303 \mathrm{~K}$ to $438 \mathrm{~K}$, the fluorescence intensity was found to decrease with a higher quenching in the $3+$ charge state compared to the $2+$ charge state (Fig. 3). This was consistent with the conformational changes resulting from greater Coulombic repulsion in the higher charge state and supported by MD simulations. Interestingly, when a salt bridge critical for solution phase stability was removed in an Asp9Asn variant of the protein disrupting the solution phase fold, the gas-phase measurements had little influence. Combined with MD simulations, it was concluded that the hydrogen bonds are more important for the stabilization of the protein in the gas phase. It was also found that the thermal unfolding data of the Trp-cage formed from purely aqueous solution and 1:1 acetonitrile:water solution were very similar, thus suggesting the similarity in gasphase structure even when the starting solution-phase structures were different. This indicates that Trp-cage refolds into a stable gas-phase conformation upon electrospray and does not have a memory of its solution-phase structure. Similar follow-up studies also established that fluorescence probes could be used to examine the biomolecular dynamics evolving with temperature, and correlations with solution-phase measurements can give a more in-depth insight into the intrinsic properties of the biomolecules.

\section{Conclusion}

The combination of mass spectrometry and fluorescence spectroscopy has the potential to answer fundamental questions about protein conformations and their dynamics. With current developments, the technical challenges in the gas-phase fluorescence measurements are being addressed both on instrumental and technical fronts. Gas-phase measurements are advancing from investigations of small dyes and peptides to relatively larger proteins. The fluorescence measurements have also been used to extract structure, conformation, and dynamics information from
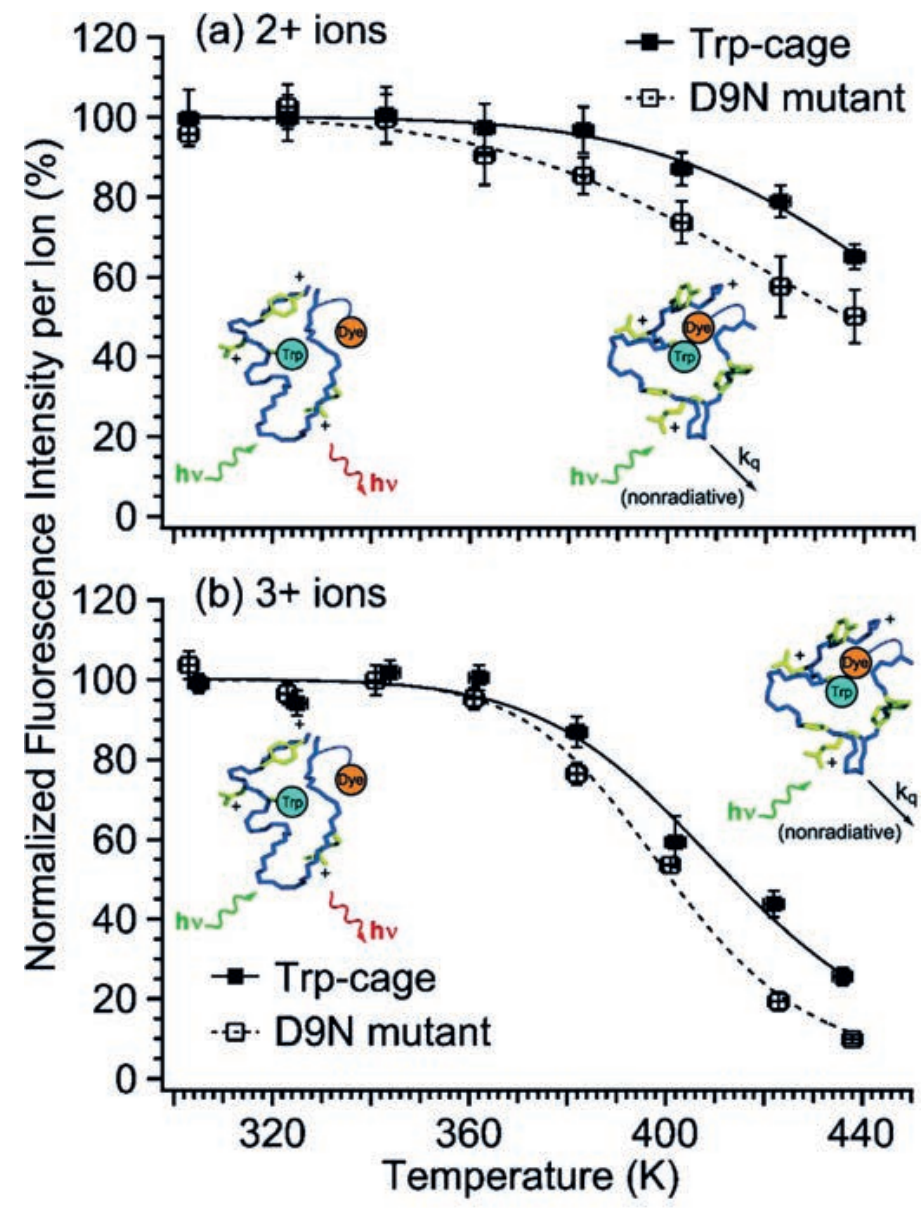

Fig. 3. Evolution of protein dynamics with temperature. Normalized fluorescence intensity per ion vs. temperature for the trapped (a) $2+$ and (b) 3+ charge state ions of desolvated Trp-cage (black square) and its Asp9Asn variant labeled with BODIPY TMR dye. The lines are model fit to the data yielding thermochemical parameters (entropy and enthalpy), which were found to be within the experimental uncertainty for Trp-cage and its mutant. The data implied that the salt bridge did not have a big role in the structural stability of the gaseous protein ions. Reproduced with permission from the publisher from ref. [3]. Copyright 2008 American Chemical Society.

the biomolecules. The application of these techniques to study large biomolecules can give insight into their intrinsic properties, their gas-phase conformations, and the influence of solvent on the behavior of a protein when compared with the solution phase measurements. To further enhance the gas-phase fluorescence measurements, different techniques, including temperature-controlled ESI source, differential mobility analyzer, and cryogenic trapping, can be coupled to better manipulate the ion population. With better chemical control, more insightful information can be extracted from the biomolecules.

\section{Acknowledgments}

The author sincerely thanks Prof. Renato Zenobi, Dr. Naresh Kumar, Neeru Mittal, and Jonas Metternich for their input and helpful discussions on the manuscript. He is also grateful to ETH Zürich for funding and to Metrohm AG and the Swiss Chemical Society for the best oral presentation award.

Received: January 30, 2021

[1] J. R. Lakowicz, 'Principles of Fluorescence Spectroscopy', 3rd Ed., 2006.

[2] A. S. Danell, J. H. Parks, Int. J. Mass Spectrom. 2003, 229, 35, https://doi.org/10.1016/S1387-3806(03)00253-7

[3] A. T. Iavarone, A. Patriksson, D. Van Der Spoel, J. H. Parks, J. Am. Chem. Soc. 2007, 129, 6726, https://doi.org/10.1021/ja065092s

[4] M. F. Czar, F. Zosel, I. König, D. Nettels, B. Wunderlich, B. Schuler, 
A. Zarrine-Afsar, R. A. Jockusch, Anal. Chem. 2015, 87, 7559, https://doi.org/10.1021/acs.analchem.5b01591

[5] F. O. Talbot, A. Rullo, H. Yao, R. A. Jockusch, J. Am. Chem. Soc. 2010, 132, 16156, https://doi.org/10.1021/ja1067405

[6] V. Frankevich, K. Barylyuk, K. Chingin, R. Nieckarz, R. Zenobi, ChemPhysChem 2013, 14, 929, https://doi.org/10.1002/cphc.201200959

[7] C. F. Karney, J. E. Ferrara, 'Modeling molecules with constraints', Technical report, Sarnoff Corp,. E-print arXiv:physics/0508116, 2005.

[8] E. J. Finehout, K. H. Lee, Biochem. Mol. Biol. Educ. 2004, 32, 93, https://doi.org/10.1002/bmb.2004.494032020331

[9] S. Mehmood, T. M. Allison, C. V. Robinson, Annu. Rev. Phys. Chem. 2015, 66, 453, https://doi.org/10.1146/annurev-physchem-040214-121732

[10] K. Rajabi, A. E. Ashcroft, S. E. Radford, Methods 2015, 89, 13, https://doi.org/10.1016/j.ymeth.2015.03.004

[11] Q. Bian, M. W. Forbes, F. O. Talbot, R. A. Jockusch, Phys. Chem. Chem. Phys. 2010, 12, 2590, https://doi.org/10.1039/B921076H

[12] H. Yao, R. A. Jockusch, J. Phys. Chem. A 2013, 117, 1351, https://doi.org/10.1021/jp309767f

[13] J. T. Khoury, S. E. Rodriguez-Cruz, J. H. Parks, J. Am. Soc. Mass Spectrom. 2002, 13, 696, https://doi.org/10.1016/S1044-0305(02)00364-1

[14] M. H. Stockett, J. Houmøller, K. Støchkel, A. Svendsen, S. Brøndsted Nielsen, Rev. Sci. Instrum. 2016, 87, 053103, https://doi.org/10.1063/1.4948316

[15] M. Dashtiev, R. Zenobi, J. Am. Soc. Mass Spectrom. 2006, 17, 855, https://doi.org/10.1016/j.jasms.2006.03.003

[16] P. Tiwari, J. B. Metternich, M. F. Czar, R. Zenobi, J. Am. Soc. Mass Spectrom. 2021, 32, 187, https://doi.org/10.1021/jasms.0c00264

[17] F. J. Grieman, B. H. Mahan, Faraday Discuss. Chem. Soc. 1981, 71, 191, https://doi.org/10.1039/DC9817100191

[18] Y. Wang, C. L. Hendrickson, A. G. Marshall, Chem. Phys. Lett. 2001, 334, 69, https://doi.org/10.1016/S0009-2614(00)01451-2

[19] B. Cage, J. Friedrich, R. B. Little, Y.-S. Wang, M. A. McFarland, C. L. Hendrickson, N. Dalal, A. G. Marshall, Chem. Phys. Lett. 2004, 394, 188, doi:10.1016/j.cplett.2004.06.119

[20] M. F. Czar, F. Zosel, I. Koenig, D. Nettels, B. Wunderlich, B. Schuler, A. Zarrine-Afsar, R. A. Jockusch, Anal. Chem. 2015, 87, 7559, https://doi.org/10.1021/acs.analchem.5b01591
[21] K. Chingin, R. M. Balabin, K. Barylyuk, H. Chen, V. Frankevich, R. Zenobi, Phys. Chem. Chem. Phys. 2010, 12, 11710, https://doi.org/10.1039/C000807A

[22] J. C. K. Kung, N. Vurgun, J. A. C. Chen, M. Nitz, R. A. Jockusch, Chem. Eur J. 2020, 26, 3479, https://doi.org/10.1002/chem.201905100

[23] M. F. Czar, R. A. Jockusch, ChemPhysChem 2013, 14, 1138, https://doi.org/10.1002/cphc.201390027

[24] V. Frankevich, V. Chagovets, F. Widjaja, K. Barylyuk, Z. Yang, R. Zenobi, Phys. Chem. Chem. Phys. 2014, 16, 8911, https://doi.org/10.1039/C3CP54521K

[25] S. Daly, F. Poussigue, A. L. Simon, L. Macaleese, F. Bertorelle, F. Chirot, R. Antoine, P. Dugourd, Anal. Chem. 2014, 86, 8798, https://doi.org/10.1021/ac502027y

[26] A. U. Petersen, C. Kjær, C. Jensen, M. Brøndsted Nielsen, S. Brøndsted Nielsen, Angew. Chemie 2020, 132, 21132, https://doi.org/10.1002/ange.202084762

[27] M. F. Czar, F. D. Breitgoff, D. Sahoo, M. Sajid, N. Ramezanian, Y. Polyhach, G. Jeschke, A. Godt, R. Zenobi, J. Phys. Chem. Lett. 2019, 10, 6942, https://doi.org/10.1021/acs.jpclett.9b02621

[28] A. T. Iavarone, D. Duft, J. H. Parks, J. Phys. Chem. A 2006, 110, 12714, https://doi.org/10.1021/jp064933e

\section{License and Terms}

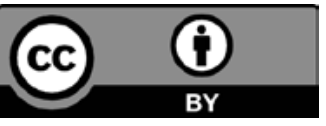

This is an Open Access article under the terms of the Creative Commons Attribution License CC BY 4.0. The material may not be used for commercial purposes.

The license is subject to the CHIMIA terms and conditions: (http:// chimia.ch/component/sppagebuilder/?view=page \&id=12).

The definitive version of this article is the electronic one that can be found at https://doi.org/10.2533/chimia.2021.252 